Pacific Journal of Mathematics

SCALAR DEPENDENT ALGEBRAS IN THE ALTERNATIVE 


\title{
SCALAR DEPENDENT ALGEBRAS IN THE ALTERNATIVE SENSE
}

\author{
JOYCE LONGMAN AND MichaEL Rich
}

Let $R$, a not necessarily associative algebra over a field $F$ of characteristic $\neq 2$, be equipped with a map $g: R \times R \times$ $R \rightarrow F$. We show that if $R$ contains a nonzero idempotent and satisfies the identities (1) $(x y) z+(y x) z=g(x, y, z)[x(y z)+$ $y(x z)]$ and $(2)(x y) z+(x z) y=g(x, y, z)[x(y z)+x(z y)]$ then $R$ is an alternative algebra. The methods also apply to other pairs of identities.

1. Introduction. In $[1,2]$ several authors have studied scalar dependent algebras, i.e., not necessarily associative algebras $R$ over a field $F$ which are equipped with a map $g: R \times R \times R \rightarrow F$ such that $(x y) z=g(x, y, z) x(y z)$ for all $x, y, z \in R$. The main result there was that if a scalar dependent algebra contains an idempotent $e$, then it is associative. In [3] the study was extended to the case of algebras over a principal ideal domain. Here we shall look at the analogous situation in the alternative case.

Specifically, suppose that $R$ is a not necessarily associative algebra over a field $F$ of characteristic $\neq 2$ equipped with a map $g: R \times R \times R \rightarrow F$ and consider the identities:

$$
\begin{aligned}
& (x y) z+(y x) z=g(x, y, z)[x(y z)+y(x z)] \\
& (x y) z+(x z) y=g(x, y, z)[x(y z)+x(z y)] \\
& (x y) z+(z y) x=g(x, y, z)[x(y z)+z(y x)] \\
& x(y z)+z(y x)=g(x, y, z)[(x y) z+(z y) x] \\
& x(y z)+x(z y)=g(x, y, z)[(x y) z+(x z) y] \\
& x(y z)+y(x z)=g(x, y, z)[(x y) z+(y x) z] .
\end{aligned}
$$

Note that if $g(x, y, z) \equiv 1$ then identities (1) and (6) each imply left alternativity, (2) and (5) each imply right alternativity and identities (3) and (4) each imply flexibility. (Recall that if $(x, y, z)$ denotes $(x y) z-x(y z)$ then an algebra $A$ is called left [right] alternative if $(x, x, y)=0[(y, x, x)=0]$ for all $x, y$ in $A$ and is alternative if it is both left and right alternative. $A$ is flexible if $(x, y, x)=0$ for all $x, y$ in $A$. A flexible left (right) alternative algebra is alternative.) The intent of this paper is to show that if $R$ contains an idempotent $e$, then any pair of identities (1)-(6) which imply alternativity when $g(x, y, z) \equiv 1$, imply alternativity in all cases. Since the methods of 
proof are similar regardless of the choice of identities, to avoid repetition we present proofs only for the case of an algebra satisfying identities (1) and (2) and describe the results for the other cases at the end. Thus, unless otherwise specified, $R$ will denote an algebra satisfying (1) and (2) over a field $F$ of characteristic $\neq 2$.

It wil be useful to note that if $\alpha=g(x, y, z)$ then (1) and (2) easily reduce to

$$
(x, y, z)+(y, x, z)=(\alpha-1)[x(y z)+y(x z)]
$$

and

$$
(x, y, z)+(x, z, y)=(\alpha-1)[x(y z)+x(z y)]
$$

2. Algebras with an identity element. In this section we assume that $R$ contains an identity element 1 .

LEMMA 1. If $R$ contains an identity element 1 and satisfies (1) then $R$ is left alternative.

Proof. Let $x, y, z \in R$ and let $\alpha=g(x, y, z), \beta=g(x+1, y, z)$, $\delta=g(x, y+1, z), \gamma=g(x+1, y+1, z)$. Then we have

$$
\begin{aligned}
(\alpha-1)[x(y z)+y(x z)] & =(x, y, z)+(y, x, z) \\
& =(x+1, y, z)+(y, x+1, z) \\
& =(\beta-1)[x(y z)+y(x z)+2 y z] .
\end{aligned}
$$

Thus,

$$
(\alpha-\beta)[x(y z)+y(x z)]=2(\beta-1) y z
$$

Similarly

$$
(\alpha-\delta)[x(y z)+y(x z)]=2(\delta-1) x z .
$$

Suppose that $(x, y, z)+(y, x, z) \neq 0$ and that $\alpha=\beta$. Then by (7) $y z=0$. If $\alpha=\delta$ then by (8) $x z=0$ from which it follows that $(x, y, z)+(y, x, z)=0$. Thus $\alpha \neq \delta$ and we get from (8)

$$
y(x z)=\delta^{\prime}(x z) \quad \text { for } \quad \delta^{\prime}=\frac{2(\delta-1)}{\alpha-\delta} \in F .
$$

In a similar fashion $(\delta-1)[x(y z)+y(x z)+2 x z]=(x, y+1, z)+$ $(y+1, x, z)=(x+1, y+1, z)+(y+1, x+1, z)=(\gamma-1)[x(y z)+$ $y(x z)+2 x z+2 y z+2 z]=(\gamma-1)[y(x z)+2 x z+2 z]$ so that

$$
(\delta-\gamma)[y(x z)+2 x z]=2(\gamma-1) z .
$$

Since $\delta=\gamma$ implies that $z=0$ we get $\delta \neq \gamma$ and $y(x z)+2 x z=\mu z$ 
for $\mu \in F$. Thus $(x+1, y+1, z)+(y+1, x+1, z)=(\gamma-1)[y(x z)+$ $2 x z+2 z]=t z$ for $t \in F$. By $(9)(x, y, z)+(y, x, z)=(\alpha-1) \delta^{\prime} x z$. Thus $x z=t^{\prime} z$ for $t^{\prime} \in F$ so that $y(x z)=t^{\prime} y z=0$. Since $y(x z)=x(y z)=0$ we arrive at $(x, y, z)+(y, x, z)=0$. Thus if $x$ and $y$ don't left alternate it follows that $\alpha \neq \beta$. Since $\alpha \neq \beta$, (7) leads to:

$$
(x, y, z)+(y, x, z)=\mu^{\prime} y z \quad \text { for } \quad \mu^{\prime} \in F .
$$

Applying the same procedure as above it follows that $g(x, y, z+1) \neq$ $g(x+1, y, z+1)$ and that

$$
(x, y, z+1)+(y, x, z+1)=\mu^{\prime \prime}(y(z+1)) \quad \text { for } \quad \mu^{\prime \prime} \in F .
$$

Combining (11) and (12) we have $\left(\mu^{\prime}-\mu^{\prime \prime}\right) y z=\mu^{\prime \prime} y$. If $\mu^{\prime}=\mu^{\prime \prime}$ it follows that $x$ and $y$ left alternate. Suppose $\mu^{\prime} \neq \mu^{\prime \prime}$, then $y z=c y$ for $c \in F$. Thus, by (11) $(x, y, z)+(y, x, z)=s y$ for $s \in F$. Analogously, $(x, y+1, z)+(y+1, x, z)=s^{\prime}(y+1)$ for $s^{\prime} \in F$. Comparing the last two equations we have $\left(s-s^{\prime}\right) y=s^{\prime} 1$. Thus, either $s^{\prime}=0$ or $y$ is a scalar multiple of the identity element. In either case $(x, y, z)+(y, x, z)=0$, so $R$ is left alternative.

LEMMA 2. If $R$ contains an identity element 1 and satisfies (2) then $R$ is right alternative.

Proof. Let $x, y, z \in R, \quad \alpha=g(x, y, z)$, and $\beta=g(x+1, y, z)$. Then $(\alpha-1)[x(y z)+x(z y)]=(x, y, z)+(x, z, y)=(x+1, y, z)+$ $(x+1, z, y)=(\beta-1)[x(y z)+x(z y)+y z+z y]$. Thus we get

$$
(\alpha-\beta)[x(y z)+x(z y)]=(\beta-1)[y z+z y] .
$$

Suppose $\alpha=\beta$. If $\beta=1$ the result follows immediately whereas if $\beta \neq 1$ then $y z+z y=0$. But in this case also $(x, y, z)+(x, z, y)=$ $(\alpha-1)[x(y z+z y)]=0$ so the result holds. It follows that $\alpha \neq \beta$. In general then, if $(a, b, c)+(a, c, b) \neq 0$ then $g(a, b, c) \neq g(a+1, b, c)$. We are left with the case $\alpha \neq \beta$. Then by (13) and (2') applied to the triple $x+1, y, z$ we have $(x, y, z)+(x, z, y)=l[z y+y z]$ for $l \in F$. Applying the same argument to the triple $x, y, z+1$, we get $(x, y, z+1)+(x, z+1, y)=l^{\prime}[z y+y z+2 y]$ so that $\left(l-l^{\prime}\right)[z y+y z]=$ $2 l^{\prime} y$. If $l=l^{\prime}$ we get right alternativity as in Lemma 1 . Thus we may assume that $z y+y z=l^{\prime \prime} y$ for $l^{\prime \prime} \in F$ so that $(x, y, z)+(x, z, y)=$ $\gamma y$ for $\gamma \in F$. Similarly $(x, y+1, z)+(x, z, y+1)=\gamma^{\prime}(y+1)$ for $\gamma^{\prime} \in F$. Setting the last two equations equal we get $\left(\gamma-\gamma^{\prime}\right)=\gamma^{\prime} 1$ and it follows that either $\gamma^{\prime}=0$ or $y$ is a scalar multiple of the identity element. Thus, in any case $(x, y, z)+(x, z, y)=0$.

Combining Lemmas 1 and 2 we have 
THEOREM 1. If $R$ contains an identity element and satisfies (1) and (2) then $R$ is an alternative algebra.

3. Algebras containing an idempotent. Henceforth, we drop the assumption that $R$ contains an identity element but assume instead that it contains a nonzero idempotent.

Lemma 3. If $R$ satisfies (1) and (2) then $(e, e, R)=(R, e, e)=$ $(e, R, e)=0$.

Proof. Let $x \in R$. By (1) $e x=\alpha e(e x)$ for $\alpha=g(e, e, x)$. Thus $(e, e, x)=(\alpha-1) e(e x)$. Similarly $(e, e, x+e)=(\beta-1)[e(e x)+e]$ for $\beta=g(e, e, x+e)$. Thus $(\alpha-\beta) e(e x)=(\beta-1) e$. Now $\alpha=\beta$ implies $\beta=1$ so that $(e, e, x)=0$. Assume $\alpha \neq \beta$. Then $e(e x)$ is a scalar multiple of $e$ so that $e x$ is a scalar multiple of $e$. Thus $(e, e, x)=$ $e x-e(e x)=0$ and we have $(e, e, R)=0$. Similarly, by (2) we get $(R, e, e)=0$.

For the last identity we first note that $(e, R, e) e=0$. For if $x \in R$ then $(e, x, e) e=[(e x) e-e(x e)] e=(e x) e-[e(x e)] e=[e(x-x e)] e$. But by (1), $[e(x-x e)] e=-[(x-x e) e] e+g(e, x-x e, e)[e((x-x e) e)+$ $(x-x e) e]=0$ by the earlier remarks. Therefore $(e, R, e) e=0 . \quad$ Now by $(1)(e, x, e)=(\mu-1)[e(x e)+x e]$ for $\mu=g(e, x, e)$ and $(e, x+e, e)=$ $(\delta-1)[e(x e)+x e+2 e]$ for $\delta=g(e, x+e, e)$. Therefore $(\mu-\delta)[e(x e)+$ $x e]=2(\delta-1) e$. If $\mu=\delta$ then $\delta=1$ so that $(e, x, e)=0$. Otherwise $e(x e)+x e$ is a scalar multiple of $e$ from which it follows that $(e, x, e)=t e$ for some $t \in F$. But $0=(e, x, e) e=t e$ so that $t=0$. Thus $(e, x, e)=0$ so that $(e, R, e)=0$.

LEMMA 4. Let $e$ be an idempotent of a ring $R$ satisfying (1). Then to each $x, y \in R$ there are elements $a, b \in F$ such that $(x, y, e)+$ $(y, x, e)=a e$ and $(x, e, y)+(e, x, y)=b e$. Similarly, if $R$ satisfies (2) there are elements $c, d \in F$ such that $(x, y, e)+(x, e, y)=c e$ and $(e, x, y)+(e, y, x)=d e$.

Proof. We prove the first identity only as the others are proved analogously. By $(1)(x, y, e)+(y, x, e)=(\alpha-1)[x(y e)+y(x e)]$ and $(x, y+e, e)+(y+e, x, e)=(\beta-1)[x(y e)+y(x e)+2 x e]$ for $\alpha=g(x, y, e)$ and $\beta=g(x, y+e, e)$. Thus, $(\alpha-\beta)[x(y e)+y(x e)]=2(\beta-1) x e$. If $\alpha \neq \beta$ then $x(y e)+y(x e)=l(x e)$ for $l \in F$. If $\alpha=\beta$ then either $\beta=1$ or $x e=0$. In any event we have $(x, y, e)+(y, x, e)=t(x e)$ for $t \in F$ or $x e=0$. Similarly $(x+e, y, e)+(y, x+e, e)=t^{\prime}(x e+e)$ for $t^{\prime} \in F$ or $x e+e=0$. A simple analysis of the four combinations yields $(x, y, e)+(y, x, e)=a e$ for some $a \in F$.

It is well known that Lemma 3 implies that, relative to an 
idempotent $e, R$ has a Peirce decomposition $R=R_{11}+R_{10}+R_{01}+R_{00}$ where $R_{i j}=\{x \in R \mid e x=i x, x e=j x\}$. Thus, we only have to prove the multiplicative properties in:

THEOREM 2. Let $R$ be an algebra satisfying (1) and (2). Then if $e$ is an idempotent of $R, R$ has a Peirce decomposition $R=R_{11}+$ $R_{10}+R_{01}+R_{00}$ relative to $e$ and the Peirce subspaces multiply according to:
(a) $R_{i j} R_{j k} \subseteq R_{i k}$.
(b) $R_{i j} R_{i j} \subseteq R_{j i}$.
(c) $R_{i j} R_{k l}=0$ if $j \neq k$ and $(i, j) \neq(k, l)$.
(d) $r_{i j}^{2}=0$ for any $r_{i j} \in R_{i j}, i \neq j$.

Proof. Let $x, y \in R_{i i}$. By Lemma $4(x, y, e)+(x, e, y)=c e$ or $(x y) e-i(x y)=c e$. If $i=1$ then by writing $x y=a_{11}+a_{10}+a_{01}+a_{00}$ and comparing component parts of both sides of the equation we get $x y \in R_{11}+R_{01}$. Thus $R_{11}^{2} \subseteq R_{11}+R_{01}$. From Lemma 4 again $(x, e, y)+(e, x, y)=b e$ or $i(x y)-e(x y)=b e$. From this we get $R_{11}^{2} \subseteq R_{11}+R_{10}$ and $R_{00}^{2} \subseteq R_{11}+R_{01}+R_{00}$. Hence $R_{11}^{2} \subseteq\left(R_{11}+R_{01}\right) \cap$ $\left(R_{11}+R_{10}\right)=R_{11}$. In (2) let $x, y \in R_{00}, z=e$ to get $(x y) e=0$. Thus $R_{00}^{2} \subseteq\left(R_{00}+R_{10}\right) \cap\left(R_{11}+R_{01}+R_{00}\right)=R_{00}$. Hence the $R_{i i}$ are subalgebras.

To show that the subalgebras are othogonal, let $x \in R_{i i}, y \in R_{j j}$, $i \neq j$. Then from $(x, y, e)+(x, e, y)=c e$, we get $(x y) e+(i-2 j) x y=$ $c e$ from which it follows that $x y=\delta e \in R_{11}$ for some $\delta \in F$. Thus $R_{i i} R_{j j} \leqq R_{11}$. Now let $x \in R_{11}, y \in R_{00}$. By (2) $(x y) e+(x e) y=\alpha[x(y e)+$ $x(e y)]=0$ so that $2 x y=x y=0$. By (1) $(y x) e=g(y, x, e)[y(x e)+x(y e)]$ and by (2) $(y x) e+(y e) x=g(y, x, e)[y(x e)+y(e x)]$. Since $y x \in R_{11}$ and $x y=0$ we arrive at $y x=g(y, x, e)(y x)=2 g(y, x, e)(y x)$. Thus $y x=0$ and we have $R_{11} R_{00}=R_{00} R_{11}=0$.

Before proceeding note that $R_{i j} R_{j j}+R_{i i} R_{i j} \cong R_{11}+R_{i j}$ for all $i, j$. For by Lemma $4,(x, e, y)+(e, x, y)=b e$. Then if $x \in R_{11}$, $y \in R_{10}$ or $x \in R_{10}, y \in R_{00}$ we obtain $x y-e(x y)=b e \in R_{11}$ so that $x y \in R_{11}+R_{10}$. Similarly in $(x, e, y)+(x, y, e)=c e$ let $x \in R_{00}, y \in R_{01}$ or $x \in R_{01}, y \in R_{11}$ to get $(x y) e-x y=c e \in R_{11}$ so that $x y \in R_{11}+R_{01}$.

Next let $x \in R_{i i}, y \in R_{i j}$. Then $x y \in R_{11}+R_{i j}$. If $\alpha=g(x, e, y)$ then by (1), $(x e) y+(e x) y=\alpha[x(e y)+e(x y)]$ is $2 i x y=\alpha[i x y+e(x y)]$. Also by (2), $(x e) y+(x y) e=\alpha[x(e y)+x(y e)]$ is $i x y+(y x) e=\alpha x y$. If $\alpha=1$ then $i x y=e(x y)$ and $i x y+(x y) e=x y$ imply $(x y)_{11}=0$ so that $x y \in R_{i j}$. If $\alpha \neq 1$ and $i=1$ then by (1) $2 x y=2 \alpha x y$ so that $x y=0$, whereas if $i=0$, (2) gives $x y=\alpha x y$ and again $x y=0$. Therefore, in all cases $x y \in R_{i j}$ or $R_{i i} R_{i j} \subseteq R_{i j}$.

By Lemma 4, $(y, x, e)+(y, e, x)=c^{\prime} e$ reduces to 


$$
(y x) e+(j-2 i) y x=c^{\prime} e
$$

and we have $y x \in R_{11}$. Next let $\mu=g(e, y, x)$. By (1), (ey) $x+(y e) x=$ $\mu[e(y x)+y(e x)]$ or $y x=\mu[y x+i y x]$. By (2), $(e y) x+(e x) y=\mu[e(y x)+$ $e(x y)]$ or $i y x+i x y=\mu[y x+i x y]$. If $i=1, y x=2 \mu y x$ and $y x+x y=$ $\mu[y x+x y]$. If $\mu \neq 1$ then $y x=-x y$ so that $y x \in R_{11} \cap R_{i j}=0$. If $\mu=1, y x=2 y x$ implies $y x=0$. If $i=0$, then $\mu y x=0$ implies $y x=0$. Hence $y x=0$ in all cases and $R_{i j} R_{i i}=0$.

Now let $x \in R_{i i}, y \in R_{j i}$ and $\nu=g(y, e, x)$. Then $y x \in R_{11}+R_{j i}$. By (1) we obtain $y x=\nu(i y x+e(y x))$ and by (2) we have $i(y x)+(y x) e=$ $2 \nu i(y x)$. Therefore, if $i=0$ we have $(y x) e=0$ so that $(y x)_{11}=0$. If $i=1$ we have $(1-\nu) y x=\nu[e(y x)]$ and $2 y x=2 \nu y x$. Therefore, if $\nu \neq 1$ then $y x=0$ whereas, if $\nu=1, e(y x)=0$ so that $(y x)_{11}=0$. Hence, in all cases $y x \in R_{j i}$ or $R_{j i} R_{i i} \subseteq R_{j i}$.

By Lemma $4,(x, e, y)+(e, x, y)=b e$ which reduces to $(3 i-1) x y-$ $e(x y)=b e$. This implies that $x y \in R_{11}$. Now let $\delta=g(x, y, e)$. Then (1) implies $x y+i(y x)=\delta i(x y+y x)$ so that if $i=0$ then $x y=0$. (2) implies $(1+i)(x y)=\delta i x y$. Therefore, if $i=1$ we obtain $x y+y x=$ $\delta(x y+y x)$ and $2 x y=\delta x y$. Therefore, if $\delta=1$ then $x y=0$ whereas, if $\delta \neq 1$ we have $x y=-y x \in R_{11} \cap R_{01}=0$. Thus $R_{i i} R_{j i}=0$.

Let $x \in R_{10}, y \in R_{01}$. Then $(x, e, y)+(e, x, y)=b e$ reduces to $x y-$ $e(x y)=b e$ so that $x y \in R_{11}+R_{10}$. Also $(x, y, e)+(x, e, y)=c e$ reduces to $(x y) e-x y=c e$ so that $x y \in R_{11}+R_{01}$. Thus $x y \in\left(R_{11}+R_{01}\right) \cap$ $\left(R_{11}+R_{10}\right)=R_{11}$ and $R_{10} R_{01} \subseteq R_{11}$. Now $(y, e, x)+(e, y, x)=b^{\prime} e$ reduces to $-e(y x)=b^{\prime} e$ so that $y x \in R_{11}+R_{01}+R_{00}$. Let $\alpha=g(x, y, e)$. Then $(x y) e+(y x) e=\alpha[x(y e)+y(x e)]$ and $(x y) e+(x e) y=\alpha[x(y e)+x(e y)]$ reduces to $x y+(y x) e=\alpha x y$ and $x y=\alpha x y$, respectively. Thus $(y x) e=0$ and $y x \in R_{00}$. Hence $R_{01} R_{10} \subseteq R_{00}$.

We have established (a) and (c). For (b) let $x, y \in R_{10}$. Then $(x, y, e)+(x, e, y)=c e$ reduces to $(x y) e-x y=c e$ so that $x y \in R_{11}+R_{01}$. Let $\delta=g(x, e, y)$. Then $(x e) y+(x y) e=\delta[x(e y)+x(y e)]$ reduces to $x y=\delta(x y)$. Thus, if $x y \neq 0$ then $\delta=1$. But then we get $(x e) y+$ $(e x) y=x(e y)+e(x y)$ or $e(x y)=0$. Thus $x y \in R_{01}$ so that $R_{10}^{2} \subseteq R_{01}$.

If $x, y \in R_{01}$, then $(x, e, y)+(e, x, y)=b e$ reduces to $x y-e(x y)=b e$ or $x y \in R_{11}+R_{01}$. Let $\delta=g(x, e, y)$. Then $(x e) y+(e x) y=\delta[x(e y)+$ $e(x y)]$ and $(x e) y+(x y) e=\delta[x(e y)+x(y e)]$ reduce to $x y=\delta x y$ and $x y+(x y) e=\delta x y$, respectively. Thus $(x y) e=0$ and $x y \in R_{10}$. Hence $R_{01}^{2} \subseteq R_{10}$.

Finally, for (d) let $x \in R_{i j}$ for $i \neq j$. Then $x^{2} \in R_{j i}$. Let $y=x$ in $(x, y, e)+(y, x, e)=c e$ to obtain $2(i-j) x^{2}=a e \in R_{11}$. Since $i \neq j$ and $x^{2} \in R_{j i}$, it follows that $x^{2}=0$.

The alternative nucleus, $N_{A}(R)$, of an arbitrary ring $R$ is defined by $N_{A}(R)=\{r \in R \mid(x, r, x)=0$ and $(r, y, x)=(y, x, r)=(x, r, y)$ for 
all $x, y \in R$. It is shown in [4] that Theorem 2 is equivalent to the fact that $e \in N_{A}(R)$ for any idempotent $e$ of $R$. It is immediate that if $i \neq j$ then $R_{i j} \subseteq N_{A}(R)$ for if $r_{i j} \in R_{i j}$ then $\left(e+r_{i j}\right)^{2}=e+r_{i j}$. Thus $e+r_{i j}$ is idempotent so that $e+r_{i j} \in N_{A}(R)$ and so $r_{i j} \in N_{A}(R)$.

Lemma 5. $R_{11}$ and $R_{00}$ are alternative subalgebras of $R$.

Proof. It is immediate from Theorem 1 that $R_{11}$ is alternative since $R_{11}$ is an algebra which contains an identity element $e$. Now let $x, y, z \in R_{00}, \alpha=g(x, y, z)$, and $\beta=g(x+e, y+e, z+e)$. Then by $\left(1^{\prime}\right)(x, y, z)+(y, x, z)=(\alpha-1)[x(y z)+y(x) z]$. Similarly

$$
\begin{gathered}
(x, y, z)+(y, x, z)=(e+x, e+y, e+z)+(e+y, e+x, e+z) \\
=(\beta-1)[(e+x)[(e+y)(e+z)]+(e+y)[(e+x)(e+z)]] .
\end{gathered}
$$

Comparing the last two identities we obtain $(\alpha-\beta)[x(y z)+y(x z)]=$ $2(\beta-1) e$ since $R_{00}$ is a subalgebra. If $\alpha=\beta$ then $\beta=1$ so that $R_{00}$ is left alternative. If $\alpha \neq \beta$ then $x(y z)+y(x z)=2[(\beta-1) /(\alpha-\beta)] e$. But $x(y z)+y(x z) \in R_{00}$ and $2[(\beta-1) /(\alpha-\beta)] e \in R_{11}$. Thus $x(y z)+y(x z)=0$ and $\beta=1$. Hence $R_{00}$ is left alternative. A similar argument using $\left(2^{\prime}\right)$ shows that $R_{00}$ is right alternative.

THEOREM 3. If $R$ is an algebra satisfying (1) and (2) and if $R$ contains a nonzero idempotent element, then $R$ is alternative.

Proof. Let $x, y \in R$. Then $x=\sum_{i, j=0}^{1} x_{i j}$ and $y=\sum_{i, j=0}^{1} y_{i j}$ so that $(x, x, y)=\sum_{i, j=0}^{1}\left(x, x, y_{i j}\right)$. Now if $i \neq j$, then $y_{i j} \in N_{A}(R)$ so that, by the definition of $N_{A}(R),\left(x, x, y_{i j}\right)=0$. Thus, $(x, x, y)$ reduces to $\sum_{l=0}^{1}\left(x, x, y_{l l}\right)=\sum_{i, j, k, r=0}^{1}\left(x_{i j}, x_{k r}, y_{l l}\right)$. Let $S$ denote the sum $\sum_{i, j, k, r, l=0}^{1}\left(x_{i j}, x_{k r}, y_{l l}\right)$. The terms in $S$ of the form $\left(x_{i j}, x_{k k}, y_{l l}\right)$ are all zero by Theorem 2 and Lemma 5 . The terms in $S$ of the form $\left(x_{i j}, x_{i j}, y_{l l}\right)$ for $i \neq j$ are all zero since $x_{i j} \in N_{A}(R)$. Finally the other terms in $S$ come in pairs of the form $\left(x_{i j}, x_{k r}, y_{l l}\right)+\left(x_{k r}, x_{i j}, y_{l l}\right)$. Since $i \neq j$ or $k \neq r$ the sum of each of these pairs is zero. Thus $(x, x, y)=0$ and $R$ is left alternative. Similarly $R$ is right alternative.

The result of Theorem 1 holds true if the ring satisfies any pair of the identities (1)-(6) other than the pairs (1) and (6), (2) and (5), and (3) and (4) for which the result is obviously not true. The same holds true if the ring does not contain an identity but does contain a nonzero idempotent, except that here the case in which the identities (1) and (5) are satisfied is left open since we are unable to establish the property $R_{00}^{2} \subseteq R_{00}$ in this case. The proofs vary somewhat from those presented here but the basic attack is the same. Detailed proofs are available from the authors upon request. 


\section{REFERENCES}

1. R. Coughlin and M. Rich, On scalar dependent algebras, Canad. J. Math., 24 (1972), 696-702.

2. R. Coughlin, E. Kleinfeld, and M. Rich, Scalar dependent algebras, Proc. Amer. Math. Soc., 39 (1973), 69-72.

3. K. Koh, J. Luh, and M. Putcha, On the associativity and commutativity of algebras over commutative rings, Pacific J. Math., 63 (1976), 423-430.

4. M. Rich, Rings with idempotents in their nucleii, Trans. Amer. Math. Soc., 208 (1975), 81-90.

Received May 3, 1977 and in revised form November 2, 1977.

VILLANOVA UNIVERSITY

VILLANOVA, PA 19085

AND

Temple University

Philadelphia, PA 19121 


\section{PACIFIC JOURNAL OF MATHEMATICS}

\section{EDITORS}

RICHARD ARENS (Managing Editor)

University of California

Los Angeles, CA 90024

Charles W. Curtis

University of Oregon

Eugene, OR 97403

C. C. MOORE

University of California

Berkeley, CA 94720

\section{J. DugundJI}

Department of Mathematics

University of Southern California

Los Angeles, CA 90007

R. FinN and J. Milgram

Stanford University

Stanford, CA 94305

\section{ASSOCIATE EDITORS}
E. F. BECKENBACH
B. H. NeumanN
F. WOLF
K. YosHIDA

\section{SUPPORTING INSTITUTIONS}

\author{
UNIVERSITY OF BRITISH COLUMBIA \\ CALIFORNIA INSTITUTE OF TECHNOLOGY \\ UNIVERSITY OF CALIFORNIA \\ MONTANA STATE UNIVERSITY \\ UNIVERSITY OF NEVADA, RENO \\ NEW MEXICO STATE UNIVERSITY \\ OREGON STATE UNIVERSITY \\ UNIVERSITY OF OREGON
}

\author{
UNIVERSITY OF SOUTHERN CALIFORNIA \\ STANFORD UNIVERSITY \\ UNIVERSITY OF HAWAII \\ UNIVERSITY OF TOKYO \\ UNIVERSITY OF UTAH \\ WASHINGTON STATE UNIVERSITY \\ UNIVERSITY OF WASHINGTON
}

The Supporting Institutions listed above contribute to the cost of publication of this Journal, but they are not owners or publishers and have no responsibility for its content or policies.

Mathematical papers intended for publication in the Pacific Journal of Mathematics should be in typed form or offset-reproduced, (not dittoed), double spaced with large margins. Please do not use built up fractions in the text of the manuscript. However, you may use them in the displayed equations. Underline Greek letters in red, German in green, and script in blue. The first paragraph or two must be capable of being used separately as a synopsis of the entire paper. Items of the bibliography should not be cited there unless absolutely necessary, in which case they must be identified by author and journal, rather than by item number. Manuscripts, in triplicate, may be sent to any one of the editors. Please classify according to the scheme of Math. Reviews, Index to Vol. 39. All other communications should be addressed to the managing editor, or Elaine Barth, University of California, Los Angeles, California, 90024.

50 reprints to each author are provided free for each article, only if page charges have been substantially paid. Additional copies may be obtained at cost in multiples of 50 .

The Pacific Journal of Mathematics is issued monthly as of January 1966. Regular subscription rate: $\$ 72.00$ a year (6 Vols., 12 issues). Special rate: $\$ 36.00$ a year to individual members of supporting institutions.

Subscriptions, orders for numbers issued in the last three calendar years, and changes of address should be sent to Pacific Journal of Mathematics, 103 Highland Boulevard, Berkeley, California, 94708. Older back numbers obtainable from Kraus Periodicals Co., Route 100, Millwood, NY 10546.

PUBLISHED BY PACIFIC JOURNAL OF MATHEMATICS, A NON-PROFIT CORPORATION

Printed at Kokusai Bunken Insatsusha (International Academic Printing Co., Ltd.). 8-8, 3-chome, Takadanobaba, Shinjuku-ku, Tokyo 160, Japan.

Copyright (C) 1978 by Pacific Journal of Mathematics Manufactured and first issued in Japan 


\section{Pacific Journal of Mathematics}

\section{Vol. 76, No. $2 \quad$ December, 1978}

Stephanie Brewster Brewer Taylor Alexander, Local and global convexity in complete Riemannian manifolds ...........................

Claudi Alsina i Català, On countable products and algebraic convexifications of probabilistic metric spaces ...............................

Joel David Berman and George Grätzer, Uniform representations of

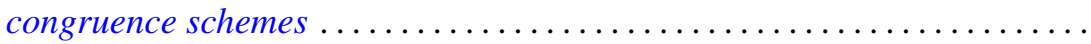

Ajit Kaur Chilana and Kenneth Allen Ross, Spectral synthesis in

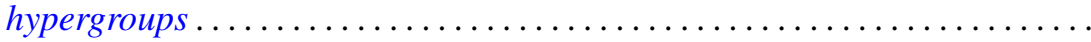

David Mordecai Cohen and Howard Leonard Resnikoff, Hermitian quadratic forms and Hermitian modular forms . .........................

Frank Rimi DeMeyer, Metabelian groups with an irreducible projective

representation of large degree .............................

Robert Ellis, The Furstenberg structure theorem .....................

Heinz W. Engl, Random fixed point theorems for multivalued mappings .......

William Andrew Ettling, On arc length sharpenings ..................

Kent Ralph Fuller and Joel K. Haack, Rings with quivers that are trees........

Kenneth R. Goodearl, Centers of regular self-injective rings ...............

John Gregory, Numerical algorithms for oscillation vectors of second order

differential equations including the Euler-Lagrange equation for

symmetric tridiagonal matrices.

Branko Grünbaum and Geoffrey Shephard, Isotoxal tilings

Myron Stanley Henry and Kenneth Leroy Wiggins, Applications of

approximation theory to differential equations with deviating

arguments

Mark Jungerman, The non-orientable genus of the n-cube .

Robert Richard Kallman, Only trivial Borel measures on $S_{\infty}$ are

quasi-invariant under automorphisms ................

Joyce Longman and Michael Rich, Scalar dependent algebras in the

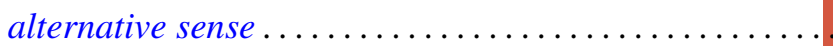

Richard A. Mollin, The Schur group of a field of characteristic zero ........ 471

David Pokrass, Some radical properties of rings with $(a, b, c)=(c, a, b) \ldots 479$

Margaret Shay and Paul Ruel Young, Characterizing the orders changed by

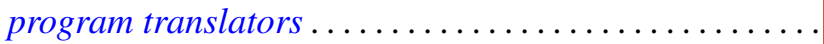

Jerrold Norman Siegel, On the structure of $B_{\infty}(F), F$ a stable space...

Surjeet Singh, (hnp)-rings over which every module admits a basic

submodule...

A. K. Snyder, Universal interpolating sets and the Nevanlinna-Pick property in

Banach spaces of functions...

Jeffrey D. Vaaler, On the metric theory of Diophantine approximation ... 